\title{
Analysis of Magnetic Properties of Iron-Resin-Ferrite Soft Magnetic Composite Materials
}

\author{
Z. BirČÁKOVÁ ${ }^{a, *}$, P. KOLlÁR ${ }^{b}$, \\ J. FÜZeR ${ }^{b}$, M. Streckova ${ }^{a}$, J. SzABÓ ${ }^{a}$, \\ R. BUREŚ $\check{S}^{a}$ AND M. FÁBEROVÁ ${ }^{a}$ \\ ${ }^{a}$ Institute of Materials Research, Slovak Academy of Sciences, \\ Watsonova 47, 04001 Košice, Slovakia \\ ${ }^{b}$ Institute of Physics, Faculty of Science, P.J. Šafárik University, \\ Park Angelinum 9, 04154 Košice, Slovakia
}

Received: 17.02.2021 \& Accepted: 19.04.2021

Doi: $10.12693 /$ APhysPolA.140.64

*e-mail: zbircakova@saske.sk

\begin{abstract}
The analysis of magnetic properties of soft magnetic composite materials composed of iron insulated by polymer bonded $\mathrm{Ni}-\mathrm{Zn}$ ferrite nanofibers is presented in the paper, focusing on the investigation of magnetization processes, by means of the total energy loss separation, the peak, differential, reversible and irreversible permeability analysis and modelling and the percent proportions of reversible and irreversible processes determination. The highest permeability exhibited by the sample with the optimal ferrite to resin ratio within insulation means the highest number of movable domain walls involved in the magnetization process. The highest proportion of irreversible domain wall displacements and the lowest proportion of reversible magnetization vector rotations were found for this sample, as well as the highest low induction losses and the lowest high induction losses, which also revealed the highest amount of domain wall displacements, facilitating magnetization reversal and resulting in the improved soft magnetic properties.
\end{abstract}

topics: soft magnetic composites, ferrite nanofibers, reversible permeability, magnetization process

\section{Introduction}

At present, scientists and engineers are constantly working on the development of new materials with the best possible magnetic properties suitable for specific applications — various chemical compositions and spectrum of parameters influencing the behavior or structure of the material are investigated as well as various preparation processes. In recent decades, the range of available soft magnetic materials has significantly expanded to include, e.g., nanocrystalline and amorphous alloys, materials prepared by compacting of ferromagnetic powders, and composite materials [1-6].

Soft magnetic composite materials (SMCs) constitute a significant subclass of soft magnetic materials due to their growing importance in electromagnetic applications as electric motors, generators, transformers, or sensors. SMCs are composed of magnetic powder particles that are electrically insulated from each other $[1,6-10]$. They provide several properties which were improved as compared to traditionally used electrical steels or soft ferrites, combining the advantages of both. SMCs exhibit three-dimensional isotropic physical properties behavior, relatively high saturation magnetic polarization, the Curie temperature and magnetic permeability and relatively low coercivity, but their main feature is that an insulating layer between conducting ferromagnetic particles minimizes the eddy current loss which results in low total power losses at elevated frequencies $\geq 100 \mathrm{~Hz}$ up to $\mathrm{MHz}$ [6-10]. In addition, the powder metallurgy techniques of composite production allow a wide optimization of the shapes of final components with a reduction in material consumption [10]. The research on SMCs has shown their vast potential in recent decades, focusing on a detailed examination of various factors influencing their properties.

It is necessary to minimize the insulation content in SMC due to the negative effect of inner demagnetizing fields [8] so that high permeability and density can be achieved, but concurrently the quality of the insulation coating of each magnetic particle must be ensured. In general, the coatings are organic, inorganic or mixed. The most commonly used organic substances are thermosets as epoxy resins, polyurethane, polyester and acrylic powders, 
and their hybrid compounds. The inorganic ones comprise, e.g. phosphates, sulfates, and various ceramics including the soft magnetic ferrites [10]. Soft magnetic ferrites promise advantageous coating not only insulating magnetic powder particles, but also ensuring magnetic flux continuity in SMC. The production of ferrite-matrix composites with $\mathrm{Fe}$ powder as the base ferromagnet faces serious technological problems [10], so the use of a binder for a fully sintered ferrite insulator seems to be a suitable solution [11].

Analyses of magnetic properties of materials provide the insight into magnetization processes behavior, whose knowledge might be important for the properties optimization [12-14]. One of many useful methods was proposed by Landgraf et al. [15-17], namely the "method of separation of total energy losses into high and low induction losses". It can be applied to both DC and AC hysteresis loops and it attempts to connect particular types of magnetization processes with the loss components. The method has yielded valuable results on various electrical steels [15, 16, 18, 19], soft ferrites [17] or composites [8]. The magnetic permeability can be defined in various ways in relation to magnetization curves. The reversible and irreversible permeability reveal the proportions of the reversible and irreversible magnetization processes along DC magnetization curves [12-14]. Important are also analytical expressions and models for magnetization curves, which enable to predict the curves and reveal various information on magnetic properties or phenomena $[1,13,14]$.

In a previous work [20], the boron-modified phenolic resin was employed to bond $\mathrm{Ni}-\mathrm{Zn}$ soft magnetic ferrite nanofibers to iron powder and the relatively high initial permeability (ranging between 80-92 at frequency $100 \mathrm{~Hz}$ ) together with relatively high frequency stability (resonance frequency $475-550 \mathrm{kHz}$ ) were found for those samples, due to sufficiently high specific electrical resistivity $(65-90 \mu \Omega \mathrm{m})$. The optimal, most effective insulation was indicated to be the volume ratio of ferrite nanofibers to the resin $\frac{1}{2}: \frac{1}{2}$ as that composite exhibited the highest initial and total permeability and lowest hysteresis, total and excess losses.

In continuation, the aim of this work is to further analyse the magnetic properties with the emphasis on the magnetization processes during magnetization reversal in order to provide more complex physical information on soft magnetic composites composed of Fe powder insulated by $\mathrm{Ni}-\mathrm{Zn}$ ferrite nanofibers bonded by boron-modified phenolic resin. The magnetic properties will be analysed by means of the separation of total energy losses into high and low induction loss components at frequency $100 \mathrm{~Hz}$, in comparison with the peak and differential permeability, and by the determination of percent proportions of reversible and irreversible magnetization processes along the initial curves. The experimental dependences of the reversible and irreversible relative permeability will be compared with the ones calculated from the analytical expressions.

\section{Experimental}

\subsection{Material characterization}

Pure iron powder (polycrystalline) Fe ABC100.30 (from Höganäs AB Sweden [21]) containing irregularly shaped particles with an average size of $\approx 100 \mu \mathrm{m}$ was chosen as the ferromagnetic component of the SMC.

The soft ferrite nanofibers with composition $\mathrm{Ni}_{0.2} \mathrm{Zn}_{0.8} \mathrm{Fe}_{2} \mathrm{O}_{4}$ were prepared by the needleless electrospinning method (Nanospider ${ }^{\mathrm{TM}}$ NS Lab, Elmarco) from the water solution of polyvinyl alcohol with metal nitrates $\mathrm{Ni}\left(\mathrm{NO}_{3}\right) 2.6 \mathrm{H}_{2} \mathrm{O}$ and $\mathrm{Zn}\left(\mathrm{NO}_{3}\right) 2.6 \mathrm{H}_{2} \mathrm{O} \cdot \mathrm{Fe}\left(\mathrm{NO}_{3}\right) 3.9 \mathrm{H}_{2} \mathrm{O}$. The process of preparation with full detail description can be found in $[20,22]$. The coercivity of ferrite nanofibers does not exceed $100 \mathrm{~A} / \mathrm{m}$ at room temperature, after the final sintering $\left(800^{\circ} \mathrm{C}, 4 \mathrm{~h}\right.$, air $)$. The nanofibers exhibit a polycrystalline spinel-type ferrite character, and the cubic fcc crystal structure corresponding to the inverse spinel was confirmed in [22]. The average diameter of fibers is $50 \mathrm{~nm}$.

As a binder of ferrite nanofibers to iron particles, the boron-modified phenolic resin (PFRB) was synthesized (from phenol, formaldehyde, ammonia, and boric acid), detailedly described in [23].

SMCs with 93 vol.\% of iron powder and 7 vol.\% of electrical insulation were prepared by uniaxial compacting followed by heat curing. The insulation consisted of ferrite nanofibers, which were bonded by the resin in volume ratios (ferrite : resin) $\frac{3}{4}: \frac{1}{4}$, $\frac{1}{2}: \frac{1}{2}$ and $\frac{1}{4}: \frac{3}{4}$. The labels and parameters of the samples and preparation conditions are given in Table I. The shape of the prepared samples was a ring (height $\approx 2 \mathrm{~mm}$, outer and inner diameter: $\approx 24 \mathrm{~mm}$ and $\approx 18 \mathrm{~mm}$ ). The preparation procedure is described in [20].

TABLE I

Composition of samples and parameters of the manufacturing procedure.

\begin{tabular}{l|c|c|c}
\hline \multicolumn{1}{c|}{ Sample } & F3/4 & F1/2 & F1/4 \\
\hline $\begin{array}{l}\text { iron powder } \\
\text { content [vol.\%] }\end{array}$ & 93 & 93 & 93 \\
\hline $\begin{array}{l}\text { electrical insulation } \\
\text { content [vol.\%] }\end{array}$ & 7 & 7 & 7 \\
\hline $\begin{array}{l}\text { ferrite nanofibers } \\
\text { content [vol.\%] }\end{array}$ & 5.25 & 3.5 & 1.75 \\
\hline PFRB resin content [vol.\%] & 1.75 & 3.5 & 5.25 \\
\hline $\begin{array}{l}\text { ferrite nanofibers to PFRB } \\
\text { resin volume ratio }\end{array}$ & $\frac{3}{4}: \frac{1}{4}$ & $\frac{1}{2}: \frac{1}{2}$ & $\frac{1}{4}: \frac{3}{4}$ \\
\hline compacting pressure [MPa] & 600 & 600 & 600 \\
\hline $\begin{array}{l}\text { temperature of heat } \\
\text { curing [ }{ }^{\circ} \mathrm{C} \text { ] }\end{array}$ & 220 & 220 & 220
\end{tabular}



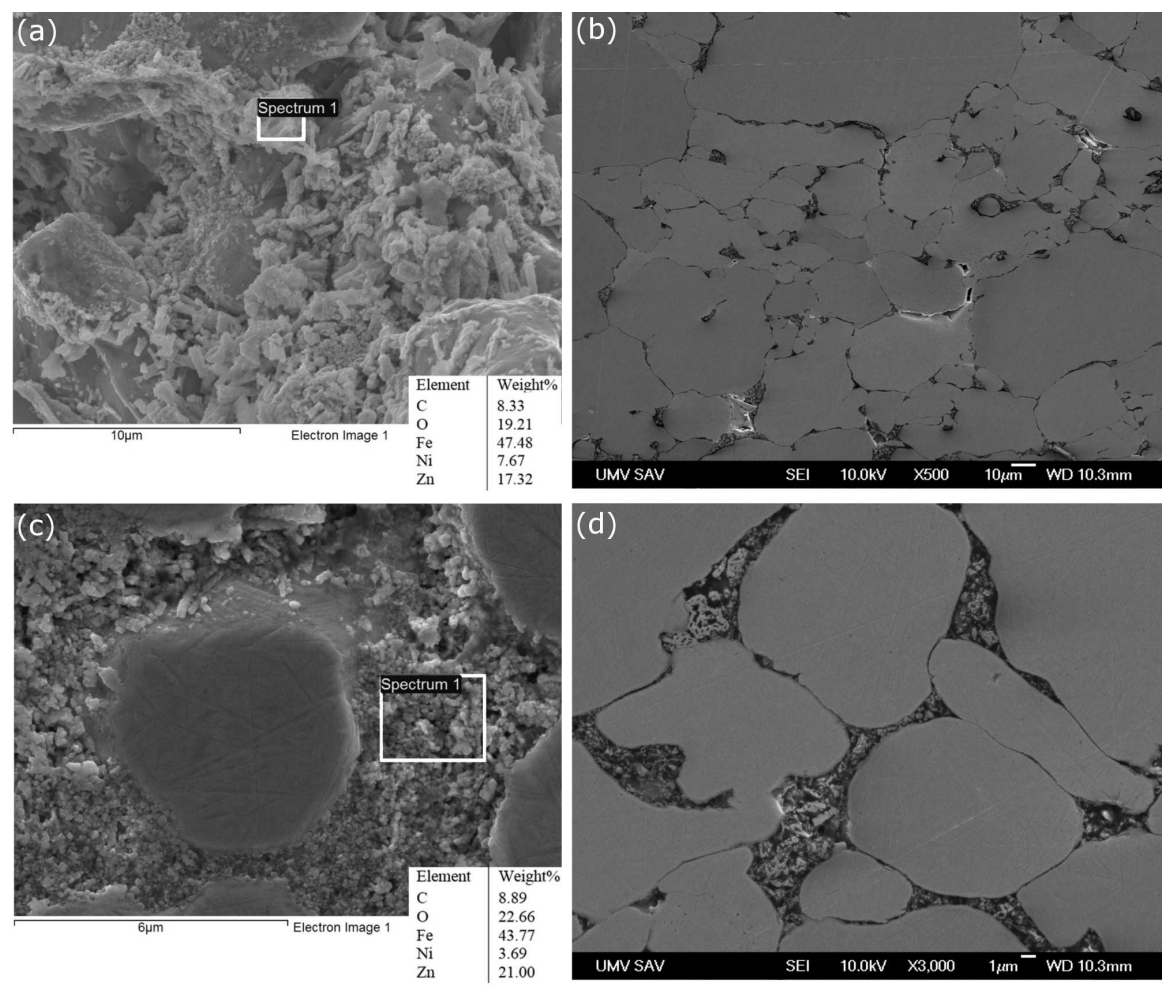

Fig. 1. SEM of sample F1/2: (a) fracture surface, showing the resin bonded ferrite nanofiber insulation of iron particles, with EDX analysis of the insulation, (b) polished surface, (c) polished surface, detail, with EDX analysis of the insulation, (d) polished surface - the detail of Fe particles coated by the resin-ferrite nanofiber mixture.

Figure 1 shows scanning electron microscopy (SEM) images of microstructures of sample F1/2. The polymer bonded ferrite nanofiber insulation of iron particles visible on the fracture surface, with results of energy dispersive X-ray spectroscopy (EDX) analysis of chemical elements of the insulation coating is depicted in Fig. 1a. The cases, e.g., the polished surface with a detailed view on iron particles insulated from each other, also with EDX analysis of the insulation, are presented in Fig. 1b-d. SEM images confirmed good coating capability of the resinferrite nanofiber mixture.

The density of samples (obtained from sample mass and dimensions) reached values between 6.75 and $7.0 \mathrm{~g} / \mathrm{cm}^{3}$ [20]. Further, the porosity was calculated using those values and the density of $\mathrm{Fe} \approx 7.86 \mathrm{~g} / \mathrm{cm}^{3}$, density of PFRB $\approx 1.14 \mathrm{~g} / \mathrm{cm}^{3}$, and density of ferrite nanofibers $\approx 4.34 \mathrm{~g} / \mathrm{cm}^{3}$. The porosity was not changing with the increasing amount of ferrite nanofibers until the ratio $\frac{1}{2}: \frac{1}{2}$ (samples $\mathrm{F} 1 / 4$ and $\mathrm{F} 1 / 2$ exhibited $6.5 \%$ of pores), but a further increase of the ferrite content at the expense of the resin resulted in the porosity increase (sample F3/4 exhibited $10.5 \%$ of pores) [20].

\subsection{Methods}

Morphology of the samples was studied by scanning electron microscope (SEM) JEOL JSM-7000F equipped with energy-dispersive $\mathrm{X}$-ray spectroscopy (EDX). The AC hysteresis loops were measured at frequency $100 \mathrm{~Hz}$ within maximum magnetic induction range from $0.05 \mathrm{~T}$ to $1.0 \mathrm{~T}$ (in ferromagnetic material) by an AC hysteresis graph MATS-2010M, on ring-shaped samples. The reversible relative permeability along the initial curves (referred to ferromagnetic content) was measured using the setup based on a lock-in amplifier reading of the induced voltage, described in $[24,25]$. The initial magnetization curves and DC hysteresis loops were measured by a DC fluxmeter-based hysteresis graph [25], up to $1.0 \mathrm{~T}$, on ring-shaped samples.

\section{Results and discussion}

In Fig. 2a, the hysteresis loops of the investigated samples measured at maximum magnetic induction $B_{m}=0.7 \mathrm{~T}$ and frequency $100 \mathrm{~Hz}$ are plotted. From each hysteresis loop, the total energy losses $W_{\text {tot }}$ can be calculated as the area of the loop, and the peak relative permeability $\mu_{\text {peak }}$ can be determined as the slope of the loop according to definition $[12,14]$ :

$$
\mu_{\text {peak }}=\frac{B_{m}}{\mu_{0} H_{m}}
$$

where $\mu_{0}$ is the vacuum permeability (also called the magnetic constant) and $H_{m}$ is the maximum magnetic field at which the maximum magnetic induction $B_{m}$ is reached during magnetization reversal. 

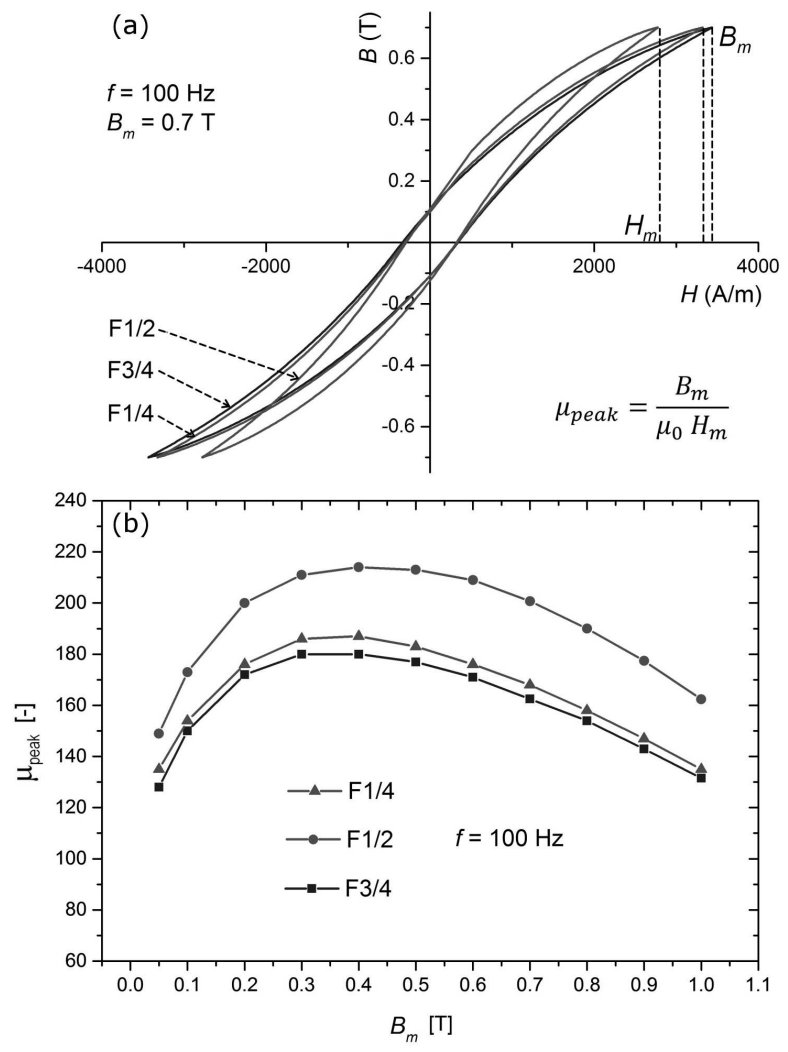

Fig. 2. (a) Hysteresis loops of the investigated samples at maximum induction $0.7 \mathrm{~T}$ and frequency $100 \mathrm{~Hz}$, with the illustration of peak permeability determination, (b) peak relative permeability of the investigated samples as a function of maximum induction in the range $0.05-1.0 \mathrm{~T}$ at frequency $100 \mathrm{~Hz}$.

The determination of $\mu_{\text {peak }}$ is illustrated in Fig. 2a, and values of $\mu_{\text {peak }}$ of the samples as a function of $B_{m}$ in the range from $0.05 \mathrm{~T}$ to $1.0 \mathrm{~T}$ at frequency $100 \mathrm{~Hz}$ are plotted in Fig. 2b. We can observe the highest peak permeability in the case of sample F1/2, which confirms the most effective ratio of soft ferrite nanofibers to PFRB resin within the insulation mixture coating Fe particles, from the view of the positive effect of magnetically active insulator vs the negative effect of increased porosity [20]. Higher permeability means that more active magnetic objects (simultaneously movable domain walls [13]) are involved in magnetization reversal and therefore more domain wall displacements occur. A higher number of movable domain walls displacing over shorter distances facilitates the magnetization reversal and leads to the magnetic properties improvement [12-14].

In Fig. 3a, the separation of total losses into high and low induction loss components is illustrated. This method is based on the statements [12-14] that a change in the dominating magnetization mechanism determining the regions of different predominant magnetization behavior at initial magnetization curve is associated with the "knee" of the initial curve, whose position is at $B_{\mu \text { max }}$ induction. This (a)
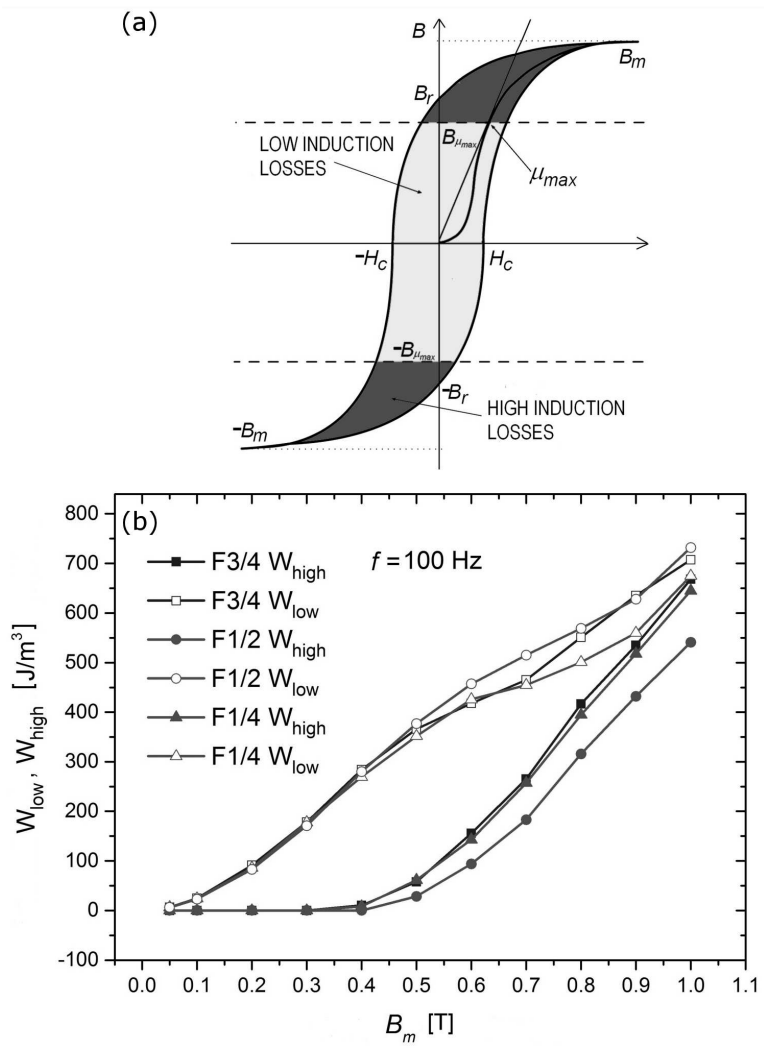

Fig. 3. (a) Illustration of the separation of total energy losses (area of hysteresis loop) into high and low induction component, (b) high and low induction energy losses of the investigated samples as a function of maximum induction in the range 0.05-1.0 $\mathrm{T}$ at frequency $100 \mathrm{~Hz}$.

point, $B_{\mu \max }$, is the point of maximum of the total relative permeability $\mu_{\text {tot }}$ (calculated at the initial curve as $\mu_{\text {tot }}=\left(B / \mu_{0} H\right)$, where $B$ is the induction and $H$ is the magnetic field $[12,14])$. The horizontal line at $B_{\mu \max }$ separates the regions of predominant magnetization processes at hysteresis loop the total energy losses $W_{\text {tot }}$ are subdivided into two parts: the high induction losses $W_{\text {high }}$ (below $B_{\mu \max }$ and above $\left.B_{\mu \max }\right)$ and the low induction losses $W_{\text {low }}$ (between $-B_{\mu \text { max }}$ and $B_{\mu \text { max }}$ ). In the high induction region, the magnetization vector rotations as well as the domain wall nucleations and annihilations dominate and in the low induction region, the dominating processes are the domain wall displacements [15-19].

In the case of $\mathrm{AC}$ magnetization, the point $B_{\mu \max }$ lies at the curve of maximum points $H_{m}, B_{m}$ of minor hysteresis loops of the same frequency, i.e., the points used for calculation of $\mu_{\text {peak }}$ with (1). The values of $B_{\mu \text { max }}$ of the investigated samples for frequency $100 \mathrm{~Hz}$ (see Fig. 2b) are given in Table II. The highest value is $0.41 \mathrm{~T}$ for sample $\mathrm{F} 1 / 2$, indicating the largest low induction region.

One can see that $W_{\text {tot }}$ at frequency $100 \mathrm{~Hz}$ were separated into $W_{\text {high }}$ and $W_{\text {low }}$ (plotted in Fig. $3 \mathrm{~b}$ ) as a function of $B_{m}$ in the range from $0.05 \mathrm{~T}$ 
TABLE II

Magnetic properties and model parameters of the samples.

\begin{tabular}{l|c|c|c}
\hline \multicolumn{1}{c|}{ Sample } & $\mathrm{F} 3 / 4$ & $\mathrm{~F} 1 / 2$ & $\mathrm{~F} 1 / 4$ \\
\hline magnetic induction $B_{\mu \max }[\mathrm{T}]$ & 0.35 & 0.41 & 0.35 \\
\hline $\begin{array}{l}\text { saturation magnetic } \\
\text { polarization } J_{S}[\mathrm{~T}]\end{array}$ & 1.803 & 1.884 & 1.877 \\
\hline parameter $a_{1}$ & 0.518 & 0.538 & 0.532 \\
\hline parameter $a_{2}$ & 1 & 1 & 1 \\
\hline parameter $C_{\text {Lin }}^{\mathrm{SMC}}$ & 1.4 & 1.3 & 1.4
\end{tabular}

to $1.0 \mathrm{~T}$. We can observe the highest $W_{\text {low }}$ and the lowest $W_{\text {high }}$ for sample $\mathrm{F} 1 / 2$, revealing the highest proportion of domain wall displacements during magnetization reversal. This is in agreement with the conclusion from peak permeability investigation that sample $\mathrm{F} 1 / 2$ contains a higher number of active magnetic objects than samples F3/4 and F1/4. It is due to the improved interactions of iron particles in SMC through the soft ferrite nanofibers acting as a magnetically active insulator mediating magnetic flux, together with the minimized amount of porosity, hence minimized the inner demagnetizing fields (arising from surface magnetic poles on insulated iron particles and ferrites [8]). This finally results in better soft magnetic properties.

In Fig. 4a, the differential, the irreversible and the reversible relative permeability along the initial magnetization curves of the investigated samples are plotted as a function of $B$ in the range from $0.05 \mathrm{~T}$ to $1.0 \mathrm{~T}$. The differential relative permeability $\mu_{\text {diff }}$ is the derivative of magnetization curve at each point $\left(H_{0}, B_{0}\right)$, reflecting the curve steepness $[12,14]$ :

$$
\mu_{\text {diff }}=\frac{1}{\mu_{0}}\left(\frac{\mathrm{d} B}{\mathrm{~d} H}\right)_{H_{0}, B_{0}} .
$$

A sum of the reversible and the irreversible relative permeability is denoted by $\mu_{\text {diff }}$ and it comprises both the reversible and the irreversible magnetization processes. The reversible relative permeability $\mu_{\text {rev }}$ is defined as follows: at the point of the initial curve $\left(H_{1}, B_{1}\right)$, a small $\mathrm{AC}$ magnetic field is superimposed and the sample is magnetized along an additional hysteresis loop, becoming a line when its amplitude $\Delta H \rightarrow 0$. Then, the slope of the line determines $\mu_{\mathrm{rev}}$, which indicates the amount of the reversible processes

$$
\mu_{\mathrm{rev}}=\frac{1}{\mu_{0}} \lim _{\Delta H \rightarrow 0}\left(\frac{\Delta B}{\Delta H}\right)_{H_{1}, B_{1}} .
$$

The irreversible relative permeability $\mu_{\mathrm{irr}}$ reflects the amount of the irreversible magnetization processes and is obtained as a difference $[12,14]$ :

$$
\mu_{\mathrm{irr}}=\mu_{\mathrm{diff}}-\mu_{\mathrm{rev}} \text {. }
$$

We can observe the highest $\mu_{\text {diff }}, \mu_{\text {irr }}$ and $\mu_{\text {rev }}$ for sample F1/2. Nevertheless, while in the case of $\mu_{\text {rev }}$ there is a minimum difference between the samples, in the case of $\mu_{\text {diff }}$ and $\mu_{\text {irr }}$ the values for
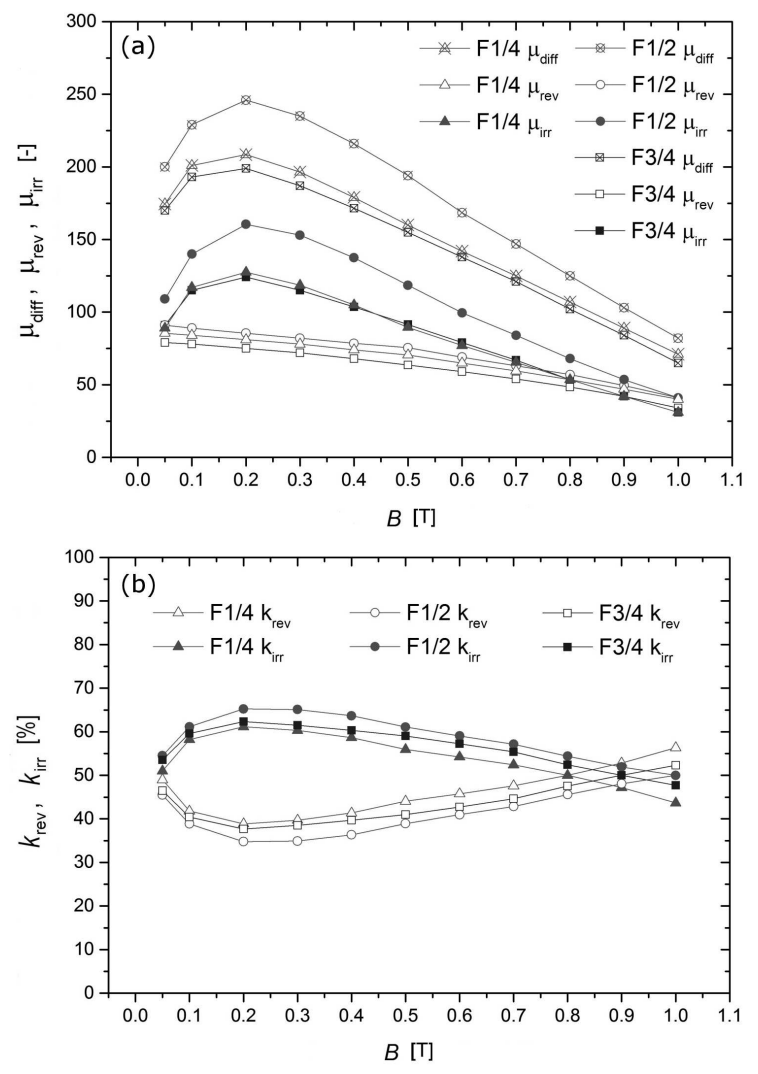

Fig. 4. (a) Differential, reversible, and irreversible relative permeability along the initial curves of the investigated samples as a function of induction in the range $0.05-1.0 \mathrm{~T}$, (b) proportions of reversible and irreversible magnetization processes along the initial curves as a function of induction in the range 0.05-1.0 T.

sample F1/2 are significantly higher. It means that a much higher amount of irreversible processes occurs during magnetizing of sample $\mathrm{F} 1 / 2$ as compared to $\mathrm{F} 1 / 4$ and $\mathrm{F} 3 / 4$, revealing a much higher number of the irreversible domain wall displacements - the Barkhausen jumps. (Domain wall displacements can be both reversible and irreversible with statistically comparable occurrence, but magnetization vector rotations are mostly reversible in the majority of soft magnetic materials [12-14].) Hence, we again found the highest number of active magnetic objects for sample $\mathrm{F} 1 / 2$, confirming the above mentioned explanations.

In Fig. 4b, the percent proportions of the reversible and the irreversible magnetization processes $\left(k_{\text {rev }}\right.$ and $\left.k_{\text {irr }}\right)$ along the initial curves of the investigated samples were determined as a function of $B$ in the range from $0.05 \mathrm{~T}$ to $1.0 \mathrm{~T}$. The proportions $k_{\text {rev }}$ and $k_{\text {irr }}$ can be calculated as

$$
\begin{aligned}
& k_{\mathrm{rev}}=\left(\frac{\mu_{\mathrm{rev}}}{\mu_{\mathrm{diff}}}\right) \times 100 \%, \\
& k_{\mathrm{irr}}=\left(\frac{\mu_{\mathrm{irr}}}{\mu_{\mathrm{diff}}}\right) \times 100 \% .
\end{aligned}
$$


The dependences of $k_{\text {rev }}$ and $k_{\text {irr }}$ vs $B$ should obey the following behavior $[12,14,16]$ : At very low $B$ (the Rayleigh region) $k_{\mathrm{rev}} \rightarrow 100 \%$, because there are only reversible domain wall displacements. Further, the Barkhausen jumps appear and their involvement in magnetization process grows until the maximum at the highest $\mu_{\text {diff }}\left(k_{\text {rev }} \rightarrow\right.$ min $)$. From the point $B_{\mu \max }$ (the highest $\mu_{\mathrm{tot}}$ ), domain wall annihilation starts to occur, thus the number of domain wall displacements (reversible as well as irreversible) starts to decrease and the magnetization process is still more realized by (reversible) magnetization vector rotations, gradually $k_{\text {rev }}$ is increasing, finally approaching saturation with $k_{\mathrm{rev}} \rightarrow 100 \%$ (no movable domain walls present to displace irreversibly). (Detailed analysis of the magnetization processes proportions in SMCs was performed in [24].) We can observe sample F1/2 exhibits the highest $k_{\text {irr }}$ and the lowest $k_{\text {rev }}$ among the investigated samples, meaning the highest proportion of irreversible domain wall displacements and the lowest proportion of reversible magnetization vector rotations, which is in agreement with all previous conclusions.

In $[26,27]$, the analytical expression for the dependence of reversible relative susceptibility $\chi_{\text {rev }}$ $\left(\chi_{\mathrm{rev}}=\mu_{\mathrm{rev}}-1\right)$ as a function of magnetization $M\left(M=\left(B / \mu_{0}\right)-H\right)$ was proposed; for structurally isotropic materials $\chi_{\text {rev }}$ was found to obey the relation

$$
\chi_{\mathrm{rev}}=\chi_{i}\left[1-\left(\frac{M}{M_{S}}\right)^{2}\right] \text {, }
$$

where $M_{S}$ is the saturation magnetization and $\chi_{i}$ is the initial susceptibility of the material. Further, different forms of the dependence were observed for different structure anisotropies. The general analytical expression involving any class of anisotropy was found to be [28]:

$$
\begin{gathered}
\frac{\chi_{\mathrm{rev}}}{\chi_{i}}=1-a_{1}\left(\frac{M}{M_{S}}\right)-a_{2}\left(\frac{M}{M_{S}}\right)^{2} \\
-\left(1-a_{1}-a_{2}\right)\left(\frac{M}{M_{S}}\right)^{4},
\end{gathered}
$$

where the structure anisotropy vector $\boldsymbol{a}=\left(a_{1}, a_{2}\right)$ is defined. The parameters $a_{1}, a_{2}$ reach values from 0 to 1 while one of them equals 1 . For predominantly anisotropic structure $a_{1}=1$ and concurrently $a_{2}$ is lower, and vice versa, for predominantly isotropic structure $a_{2}=1$ and concurrently $a_{1}$ is lower. For example, $\boldsymbol{a}=(1,0)$ was found for the hardened steel 1018 with significant anisotropy, and $\boldsymbol{a}=(0,1)$ was found for perfectly isotropic nickel-based annealed steel [28]. Using (8), we get $\chi_{\mathrm{rev}}=\chi_{i}$ for $M \rightarrow 0$ and $\chi_{\text {rev }}=0$ for $M=M_{S}$ (saturation state). Figure 5a shows the experimental dependences of $\mu_{\text {rev }}$ along the initial curves of the investigated samples as a function of $B$ in the range from $0.05 \mathrm{~T}$ to $1.0 \mathrm{~T}$. The dependences were allowed to be expressed by (8), generally valid for
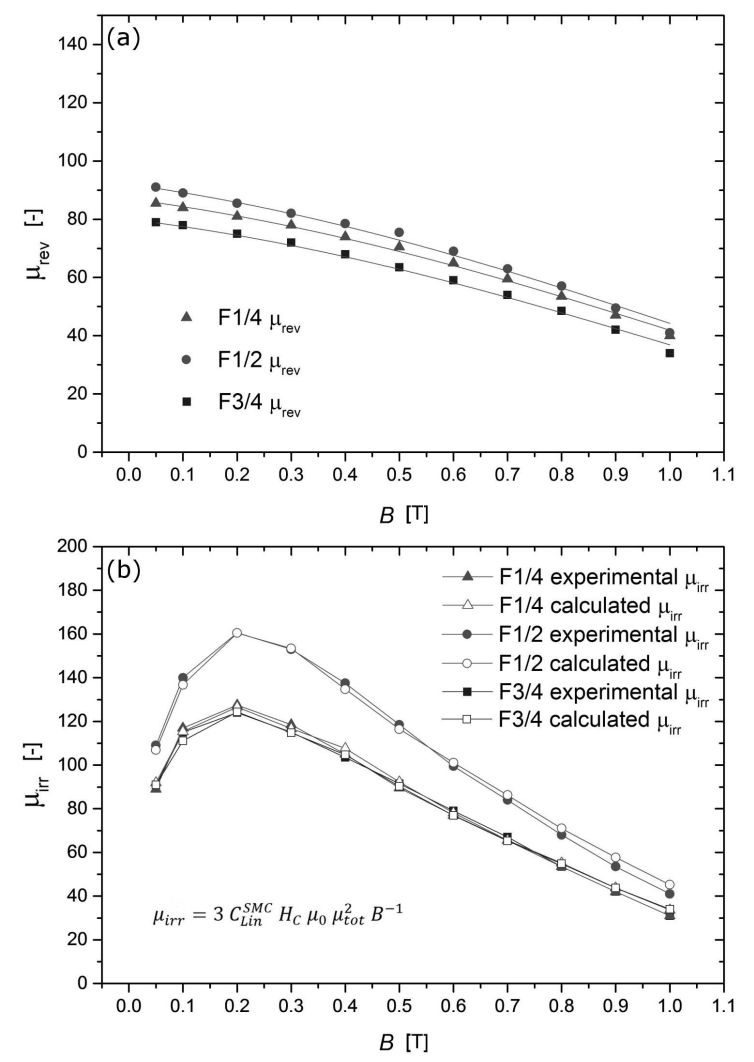

Fig. 5. (a) Experimental dependences of the reversible relative permeability along the initial curves as a function of induction, fitted with the analytical expression, (b) comparison of experimental data of the irreversible relative permeability along the initial curves as a function of induction with the ones calculated from the analytical expression.

any structural anisotropy, the fitting was performed using relations $\mu_{\mathrm{rev}}=\chi_{\mathrm{rev}}+1, \mu_{i}=\chi_{i}+1$ and approximate equality of ratios $\left(M / M_{S}\right) \approx\left(B / J_{S}\right)$. The saturation magnetic polarization $J_{S}$ (Table II) was calculated as [29]:

$$
J_{S}=\sum J_{S(x)} C_{(x)},
$$

where $C$ is the filling factor - the volume fraction of a ferromagnetic or ferrimagnetic component (excluding porosity), $J_{S(\mathrm{Fe})} \approx 2.15 \mathrm{~T}$ and $J_{S \text { (ferrite) }} \approx 0.4 \mathrm{~T}[1]$. As the structure of SMCs is supposed to be rather isotropic, the parameter $a_{2}$ was fixed to be equal to 1 during fitting. It was found that the parameter $a_{1}$ showed values about 0.5 for all investigated samples (Table II), revealing slight anisotropy due to the uniaxial pressure applied when compacting by a press. Detailed information on the optimal parameter values of the fitting is given in Table III. The parameters are unitless and, as defined with mathematical statistics, they confirm the goodness of the fitting: the reduced $\chi$ (mean squared weighted deviation) is minimized and $R$-squared (determining the strength of independent and dependent variable relationship) is close to 1 . 
Detailed information on optimal fitting values of parameters $a_{1}, a_{2}$, analyzed for all samples.

TABLE III

\begin{tabular}{c|c|c|c|c|c|c|c}
\hline \hline & Fitting equation & Reduced $\chi$ & $\begin{array}{c}\text { Adj. } \\
R \text {-squared }\end{array}$ & Value & $\begin{array}{c}\text { Std. } \\
\text { error }\end{array}$ & Value & $\begin{array}{c}\text { Std. } \\
\text { error }\end{array}$ \\
\hline F1/2 & $91\left(1-a_{1} \frac{x}{1.884}-a_{2}\left(\frac{x}{1.884}\right)^{2}-\left(1-a_{1}-a_{2}\right)\left(\frac{x}{1.884}\right)^{4}\right)+1$ & 2.23313 & 0.9919 & 0.538 & 0.01722 & 1 & 0 \\
F1/4 & $86\left(1-a_{1} \frac{x}{1.877}-a_{2}\left(\frac{x}{1.877}\right)^{2}-\left(1-a_{1}-a_{2}\right)\left(\frac{x}{1.877}\right)^{4}\right)+1$ & 0.85597 & 0.9964 & 0.532 & 0.01125 & 1 & 0 \\
F3/4 & $79\left(1-a_{1} \frac{x}{1.803}-a_{2}\left(\frac{x}{1.803}\right)^{2}-\left(1-a_{1}-a_{2}\right)\left(\frac{x}{1.803}\right)^{4}\right)+1$ & 1.30344 & 0.99429 & 0.518 & 0.01469 & 1 & 0
\end{tabular}

In previous papers $[25,30]$, the relations for the irreversible relative permeability along the initial curve were proposed for selected soft magnetic materials including SMCs, based on the expressions for DC energy losses. For Fe-phenolphormaldehyde resin SMCs, the best fitting relation was the one derived based on the linear functions approximation of DC losses [30], in the form

$$
\mu_{\mathrm{irr}}=3 \mu_{0} \mu_{\mathrm{tot}}^{2} C_{\mathrm{Lin}}^{\mathrm{SMC}} H_{C} / B,
$$

where $H_{C}$ is the coercive field of minor DC hysteresis loop of which the maximum induction point concurrently lies on the initial curve, and $C_{\mathrm{Lin}}^{\mathrm{SMC}}$ is the parameter which was found to be a constant for SMCs investigated in [30]. In Fig. 5b, the experimental data of $\mu_{\text {irr }}$ along the initial curves as a function of $B$ in the range from $0.05 \mathrm{~T}$ to $1.0 \mathrm{~T}$ are compared with the ones calculated using (10). The dependences exhibit good agreement (relative standard deviation less than $5 \%$ ), hence we have confirmed the validity of the relation (10) for another type of SMCs. The values of parameter $C_{\mathrm{Lin}}^{\mathrm{SMC}}$ are presented in Table II.

\section{Conclusion}

Magnetic properties and magnetization process of soft magnetic composites consisting of polycrystalline iron powder insulated by $\mathrm{Ni}-\mathrm{Zn}$ ferrite nanofibers bonded with boron-modified phenolic resin were analysed, especially the reversible and irreversible permeability and processes. Scanning electron microscopy documented good insulation of iron particles with the polymer-ferrite nanofiber mixture. The highest peak, the differential, reversible and irreversible relative permeability of the sample with the optimal ratio of ferrite to polymer confirmed the most effective insulation coating in terms of the positive effect of soft ferrite vs. negative effect of porosity and indicated the highest number of active magnetic objects (simultaneously movable domain walls) involved in the magnetization process. This was consistent with the observation from total energy loss separation, which revealed the highest low induction losses and the lowest high induction losses for the optimal sample, meaning the highest proportion of domain wall displacements (over shorter distances), facilitating magnetization reversal. Furthermore, this result was supported by the highest percentage of irreversible domain wall displacements and the lowest percentage of reversible magnetization vector rotations calculated for this sample. Improved interactions of iron particles through soft ferrite nanofibers along with minimized porosity have led to improved soft magnetic properties. The comparison of experimental data of the irreversible and the reversible relative permeability with the ones calculated from the analytical expressions confirmed the validity of the recently proposed relation for this type of composites and revealed their slight uniaxial anisotropy, respectively.

\section{Acknowledgments}

This work was realized within the framework of the project "MACOMA" financed by the Slovak Research and Development Agency under the contract APVV-15-0115; and by the Scientific Grant Agency of the Ministry of Education of the Slovak Republic and the Slovak Academy of Science - projects VEGA 1/0143/20, VEGA 1/0225/20 and VEGA 2/0029/21. The work was also supported by the Development Operational Program Research and Innovation for the project "New unconventional magnetic materials for applications", ITMS: 313011T544, co-funded by the European Regional Development Fund; and by the project "Research Centre of Advanced Materials and Technologies for Recent and Future Applications PROMATECH" ITMS: 26220220186, financed by the Operational Program Research and Development financed through the European Regional Development Fund.

\section{References}

[1] K.H.J. Buschow, Concise Encyclopedia of Magnetic and Superconducting Materials, Elsevier, Oxford, UK 2005.

[2] S. Atalay, O.O. Inan, V.S. Kolat, T. Izgi, Acta Phys. Pol. A 139), 159 (2021).

[3] H. Yaşar Ocak, G. Dikici Yıldız, Y. Göktürk Yıldız, B. Saatçi, R. Başar, G. Sarığlu, Acta Phys. Pol. A 139, 20 (2021).

[4] C. Liu, A. Inoue, F.L. Kong, E. Zanaeva, A. Bazlov, A. Churyumov, S.L. Zhu, F. Al-Marzouki, R.D. Shull, J. Non-Cryst. Solids 554, 120606 (2021). 
[5] O. Yalçın, H. Bayrakdar, S. Özüm, Acta Phys. Pol. A 139, 31 (2021).

[6] P. Cao, Y. Liu, J. Li, J. Du, R. Wang, T. Zhou, IEEE Trans. Magn. 57, 1 (2021).

[7] B. Kocsis, I. Fekete, I. Hatosa, L.K. Varga, Acta Phys. Pol. A 137, 886 (2020).

[8] E.A. Périgo, B. Weidenfeller, P. Kollár, J. Füzer, Appl. Phys. Rev. 5, 031301 (2018).

[9] B. Jankowski, B. Slusarek, J. Szczyglowski, K. Chwastek, Acta Phys. Pol. A 128, 116 (2015).

[10] K.J. Sunday, M.L. Taheri, Met. Pow. Rep. $72,(2017)$.

[11] W. Li, W. Wang, J. Lv, Y. Ying, J. Yu, J. Zheng, L. Qiao, S. Che, J. Magn. Magn. Mater. 456, 333 (2018).

[12] E. Kneller, Ferromagnetismus, SpringerVerlag, Berlin 1962 (in German).

[13] G. Bertotti, Hysteresis in Magnetism, Academic Press, New York 1998.

[14] S. Chikazumi, Physics of Ferromagnetism, 2nd ed., Oxford University Press, New York 1999.

[15] F.J.G. Landgraf, J.C. Teixeira, M. Emura, M.F. de Campos, C.S. Muranaka, Mater. Sci. Forum 440, 302 (1999).

[16] F.J.G. Landgraf, M.F. de Campos, J. Leicht, J. Magn. Magn. Mater. 320, 2494 (2008).

[17] F.J.G. Landgraf, V.A. Lázaro-Colán, S.R. Janasi, J. Leicht, IEEE Trans. Magn. 48, 1570 (2012).
[18] J. Leicht, N.A. Castro, E.C. Silva, F.J.G. Landgraf, A.J. Moses, T. Yonamine, J. Magn. Magn. Mater. 320, 385 (2008).

[19] B. Weidenfeller, M. Anhalt, J. Magn. Magn. Mater. 322, 696 (2010).

[20] Z. Birčáková, J. Füzer, P. Kollár, M. Streckova, J. Szabó, R. Bureš, M. Fáberová, J. Magn. Magn. Mater. 485, 1 (2019).

[21] hoganas.com.

[22] M. Strečková, E. Múdra, M. Šebek, T. Sopčák, J. Dusza, J. Kováč, Acta Phys. Pol. A 131, 729 (2017).

[23] M. Strečková, R. Bureš, M. Fáberová, L. Medvecký, J. Füzer, P. Kollár, Chin. J. Chem. Eng. 23, 736 (2015).

[24] Z. Birčáková, P. Kollár, M. Jakubčin, J. Füzer, R. Bureš, M. Fáberová, J. Magn. Magn. Mater. 483, 183 (2019).

[25] Z. Birčáková, P. Kollár, J. Füzer, R. Bureš, M. Fáberová, J. Phys. D Appl. Phys. 51, 395002 (2018).

[26] C.S. Schneider, J. Appl. Phys. 89, 1281 (2001).

[27] C.S. Schneider, IEEE Trans. Magn. 48, 3371 (2012).

[28] C.S. Schneider, J. Appl. Phys. 91, 7637 (2002).

[29] M. Anhalt, B. Weidenfeller, J. Appl. Phys. 105, 113903 (2009).

[30] Z. Birčáková, P. Kollár, J. Füzer, R. Bureš, M. Fáberová, Acta Phys. Pol. A 137, 843 (2020). 